\title{
Microstructure of nanocrystalline diamond powders studied by powder diffractometry
}

\author{
B. Palosz and E. Grzanka \\ High Pressure Research Center, Unipress, Sokolowska 29, 01142 Warsaw, Poland \\ C. Pantea, T. W. Zerda, ${ }^{\text {a) }}$ and Y. Wang \\ TCU, Physics Department, Fort Worth, Texas 76129 USA \\ J. Gubicza and T. Ungár \\ Eotvos University, Department of General Physics, P.O. Box 32, Budapest, Hungary
}

(Received 14 September 2004; accepted 4 January 2005)

\begin{abstract}
High resolution $\mathrm{x}$-ray diffraction peaks of diamond nanosize powders of nominal sizes ranging from 5 to $250 \mathrm{~nm}$ were analyzed and provided information on grain structure, average size of crystallites, and concentration of dislocations. Selected samples were heat treated at $1670 \mathrm{~K}$ at pressures 2.0 and $5.5 \mathrm{GPa}$ or had surface modified by outgassing, heat treatment at vacuum conditions, and by controlled adsorption of gases. The apparent lattice parameter method was applied to characterize the structure of a shell-core model of nanosize particles. The multiple whole profile fitting provided information on crystallite sizes and density of dislocations. Population of dislocations increased with applied pressure, while strain and interplanar distances in the surface layers decreased. Adsorption of foreign gases on the grain surface modified the structure of the surface layers but did not affect dislocations near the center of the grains. (C) 2005 American Institute of Physics.
\end{abstract}

[DOI: $10.1063 / 1.1863459]$

\section{INTRODUCTION}

Superior performance of many nanocrystalline materials has generated a lot of attention due to possible practical applications. ${ }^{1-3}$ Many experimental and theoretical studies on the structure and properties of nanosize crystals, such as diamond, $\mathrm{Si}, \mathrm{SiC}, \mathrm{Fe}, \mathrm{CdSe}, \mathrm{GaN}$, and $\mathrm{ZnO}_{2}$, have been reported. ${ }^{3-8}$ The properties of nanocrystals depend on their three-dimensional structure. Because a large fraction of atoms lies on the surface, the structure of the surface of a nanoparticle is of primary importance. The arrangement of atoms near the surface is different from that in the bulk of the nanosize crystal.This difference which has a significant effect on overall properties of the material increases with decreasing size of the nanocrystals. Due to lack of experimental data the structure of the surface layer, the so-called shell, is not fully understood. Extensive theoretical studies indicated that a short-range order dominates the structure of that layer and both disordered and ordered models of the surface component have been suggested. ${ }^{7}$

Recently Palosz and coworkers have developed a methodology of elaborating powder diffractograms of nanosize materials in terms of two distinct phases-the core and the shell components. ${ }^{9-11}$ In this model, the apparent lattice parameter model alp, both phases are ordered and the core structure is assumed to be similar to that of a large crystal. The methodology, discussed in details in previous publications, is based on a comparison of experimental results with those obtained theoretically for different models of nanosize grains. The structure of the shell has been successfully analyzed for a number of nanosize materials, including dia-

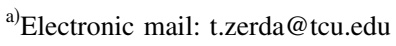

mond, $\mathrm{SiC}$ and $\mathrm{GaN}$. For diamond nanocrystals we have found that the thickness of the surface shell is between 0.5 and $0.7 \mathrm{~nm}$, with a compressive strain $1 \%$ to $3 \%$ in the shell of an untreated nanocrystalline powder. We examined also the effects of heat treatment, and gas adsorption and found that outgassing leads to a strong decrease of the strain at the surface while adsorption of gases results in the reversed effect. $^{11}$

The structure of the shell determines many mechanical properties of composites. For example, fracture toughness of nanocomposites depends on the grain boundary sliding mechanism, which is primarily defined by the structure of the grains interface. ${ }^{1,12,13}$ Dislocations, their nature and population, are other important factors that define mechanical properties of materials. ${ }^{1,7,13}$ A study of dislocations in crystals may provide valuable information on defects location, their character, and how they affect hardness, fracture toughness, and other properties of nanosize materials.

Profile analysis of $\mathrm{x}$-ray diffraction pattern has been widely used for evaluation of crystal sizes and structure of defects. Ungar and coworkers ${ }^{14,16}$ and Pantea et al. ${ }^{15}$ have developed a procedure in which Fourier coefficients of experimentally determined peak profiles are fitted by Fourier transforms of ab initio size and dislocation profiles. In the present case this method is applied to study the effect of crystal size, surface conditions, and high-pressure, hightemperature treatments on the population of dislocations in nanosize diamond crystals.

The two different diffraction methods, $\mathrm{x}$-ray alp and line profile analysis are complementary in the sense that they test homogeneous and inhomogeneous strains, respectively. The method of alp measures line shifts which are directly related to changes in average lattice spacings. X-ray line broaden- 
TABLE I. Samples of diamond powder used in this study. The average sizes obtained by the centrifuge method were provided by the manufacturer. A: Ultradiamond 90, B: Microdiamant 0-0.05, C: Microdiamant 0-0.1, D: Microdiamant $0-0.5$. The BET and centrifuge data are accurate within $15 \%$.

\begin{tabular}{lcccc}
\hline \hline & & \multicolumn{3}{c}{ Average grain size [nm] } \\
Sample & Nominal distribution of sizes $(\mathrm{nm})$ & Centrifuge method & BET & X-ray $\langle x\rangle_{\text {vol }}$ \\
\hline A & 5 & 5 & 5 & $4(1)$ \\
B & $0-50$ & 32 & 11 & $16(3)$ \\
C & $0-100$ & 50 & 18 & $24(3)$ \\
D & $0-500$ & 210 & 240 & $66(8)$ \\
\hline \hline
\end{tabular}

ing, however, is caused by fluctuation of lattice spacings. This means that any kind of homogeneous strain can be measured by methods like alp, whereas, the inhomogeneity of strain fields corresponding to lattice imperfections can be determined by the line profile analysis. A combination of the two different $\mathrm{X}$-ray diffraction methods provides complementary and detailed physical insight into the stress and strain states existing in the core and the surface regions of diamond nanoparticles treated at different temperatures under different pressure and atmospheric conditions.

\section{EXPERIMENT}

\section{A. Sample preparation}

Samples of synthetic diamond were obtained from MicroDiamant, Switzerland. Their nominal sizes are given in Table I. Sample A was studied as received and after outgassing for $20 \mathrm{~h}$ at a vacuum better than $6 \times 10^{-6}$ Torr at room temperature and at $750 \mathrm{~K}$. After outgassing, the diamond powder was exposed to different gasses (nitrogen, air, and water vapor) and the specimens were sealed in quartz tubes of very thin walls for future x-ray diffraction studies. Sample $\mathrm{B}$ was compressed at 2.0 and $5.5 \mathrm{GPa}$ at $1670 \mathrm{~K}$. The high pressure, high temperature (HPHT) experiments were run on a 250 ton hydraulic press. Two different high pressure apparatuses were utilized. A cylinder-piston-type cell was used at $2.0 \mathrm{GPa}$. For experiments at $5.5 \mathrm{GPa}$ we used a toroid cell. The pressure was stable within $\pm 2 \%$. All experiments were run at the same temperature, $T=1670 \mathrm{~K}$. A $\mathrm{W} 3 \% \mathrm{Re} / \mathrm{W} 25 \%$ Re thermocouple was used to measure and control the temperature inside the cylinder-piston cell with precision of $20 \mathrm{~K}^{17}$ For the toroid cell the temperature control was not better than $50 \mathrm{~K}$. In this paper, the compressed samples are called B2 (2 GPa) and B5.5 (5.5 GPa).

In the case of experiments in the cylinder cell we used talc tubes for thermal and electrical isolation and as holders for diamond powder. To minimize graphitization of nanodiamonds, we used water-free talc. Water acts as a catalyst for the graphitization process. ${ }^{17}$ The water-free talc was obtained from raw talc by heating for $30 \mathrm{~min}$ at $1100 \mathrm{~K}$. The heattreated talc did not release water during subsequent heating.

The toroid high pressure cell consisted of two identical anvils with toroidal grooves and the lithographic stone gasket, matched the contours of the grooves. ${ }^{18}$ The diamond powder packed inside a cylindrical graphite heater was placed inside a hole in the gasket. ${ }^{18}$ In these experiments there was no graphitization catalyst present near the diamond samples, moreover, experiments were conducted at pressures at which diamond is the stable form of carbon.

The experiments were run according to the following protocol. In the first step, at room temperature, the pressure was raised to the desired value. Next, temperature was increased to $1670 \mathrm{~K}$ at a rate of $2000 \mathrm{~K} / \mathrm{min}$. The samples were kept at that temperature for $60 \mathrm{~s}$ and then the heating was stopped and the pressure released.

Nitrogen gas adsorption analysis was run on Autosorb made by Quantasorb, $\square, \square$. The particle size was estimated using the following expression:

$$
d_{\mathrm{BET}}=6 /\left(S \rho_{0}\right),
$$

where $S$ is the nitrogen surface area obtained by the (BET) method and $\rho_{0}$ is the bulk density of diamond. The above formula is correct only for spherical particles.

\section{B. X-ray diffraction experiments}

High-resolution powder diffraction data were collected on the X3B1 beamline at the National Synchrotron Light Source, Brookhaven National Laboratory, $\square, \square$. The powders were mounted on a single-crystal quartz holder. A double $\mathrm{Si}(111)$ crystal monochromator was used to obtain a monochromatic beam of 0.69960(1) $\AA$ wavelength, as determined from seven well-defined reflections of an $\mathrm{Al}_{2} \mathrm{O}_{3}$ flat plate standard (NIST 1976a).

Before reaching the sample, the incident beam was monitored by an ion chamber and the diffracted signal was normalized for the decay of the primary beam. The diffracted beam was reflected by a $\mathrm{Ge}(111)$ analyzer crystal before being detected by a NaI scintillation counter. Diffraction data were collected at room temperature by counting for 1 to $20 \mathrm{~s}$ at each $2 \theta$, in steps of $0.02^{\circ}$ from 17.5 to $92^{\circ}$. A total of 12 diffraction peaks of diamond were recorded. As an example, in Fig. 1 we show the X-ray diffractogram for specimen B, pressure treated at $5.5 \mathrm{GPa}$.

\section{X-RAY DIFFRACTION ANALYSIS}

\section{A. Multiple whole profile fitting}

The evaluation of the peak profiles was performed by the multiple whole profile fitting (MWP) procedure. ${ }^{14}$ In this method the Fourier coefficients of the measured profiles were fitted by theoretical functions calculated on the basis of the model of the microstructure. In this model the crystallites were assumed to have spherical form with log-normal size distribution and the strains were assumed to be caused by 


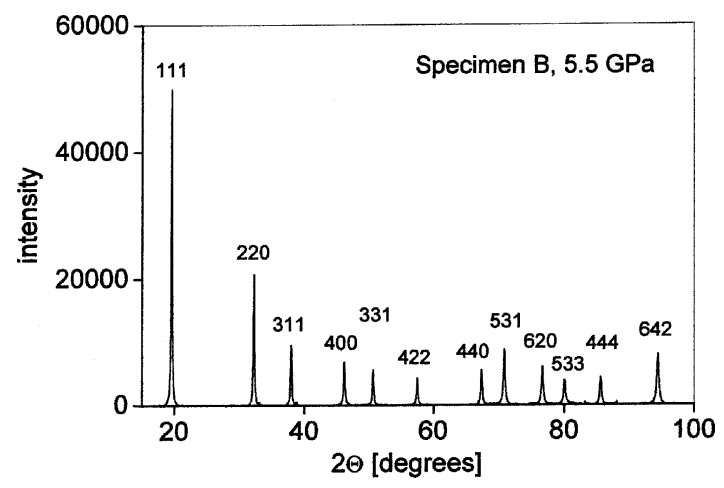

FIG. 1. X-ray diffractogram for specimen B, pressure treated at 5.5 GPa.

dislocations. Details of the method have been published previously and can be found in Refs 14-16. The dislocation model of strains is always justified when the mean square strain $\left\langle\varepsilon^{2}\right\rangle$ is a monotonously decreasing function of the Fourier variable. ${ }^{19,20}$ Since this assumption applies to the present case, we used the dislocation model of strains. Example of the quality of the fitting procedure is depicted in Fig. 2 for specimen B compressed at 5.5 GPa. The open circles and the solid lines represent the measured and calculated Fourier transforms. The differences between measured and calculated values are also shown in that figure.

As a result of the MWP fitting procedure the median $(m)$ and the variance $(\sigma)$ of the size distribution, and the density $(\rho)$ of dislocations were obtained. Parameters $m$ and $\sigma$ were used to calculate the volume weighted mean crystallite size

$$
\langle X\rangle_{\mathrm{vol}}=m \exp \left(3.5 \sigma^{2}\right) \text {. }
$$

\section{B. alp ANALYSIS}

Elaboration of a powder diffraction experiments was based on determination of positions of individual Bragg reflections. In nanosize grains, the long-range order is limited by the size of the crystallite which is smaller than the coherence length of the scattered beam and, therefore, the Bragg equation which works properly for large volume crystals, may not be applicable. ${ }^{21}$ Thus, the positions of the Bragg reflections are not determined by the unit cell parameters alone but become strongly influenced by the grain size and

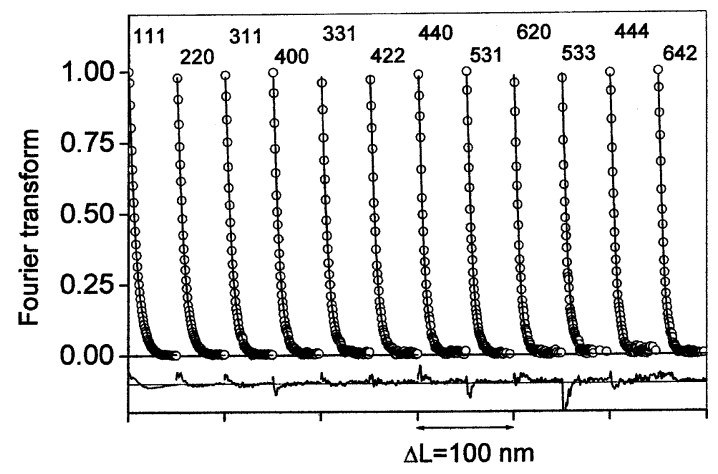

FIG. 2. MWP fitting corresponding to the specimen B, pressure treated at 5.5 GPa. The open circles and the solid lines represent the measured and the calculated Fourier transforms, respectively. The difference between the measured and the calculated values are also shown in the bottom of the figure. shape. $^{22,23}$ Departure of position of a given diffraction peak $h k l$ is caused by a short length of a Fourier series over the row of atoms in the direction normal to the $h \mathrm{kl}$ plane. Presence of strains leads either to elongation or shortening of the interatomic bonds relative to the relaxed lattice. As a result, a nanocrystal cannot be considered to be a structurally uniform object. Consequently, its structure cannot be characterized by one set of lattice parameters. The values of lattice parameters calculated from individual Bragg reflections are different and we name them "apparent lattice parameters," alp's. They are linked to the $Q$ values of the corresponding reflections $(Q$ $=4 \pi \sin \theta / \lambda$ ). No simple analytical function describing dependence of $a l p$ values on the $Q$ vector exists, therefore, we make evaluation of the crystal structure based on a comparison of the experimental and theoretical alp- $Q$ relations. For the structure with strained surface shell the alp- $Q$ relation shows a complex dependence with some characteristic minima and maxima, Figs. 4 and 5. These features can be used for identification and evaluation of the surface structure: (i) relative difference between alp values measured at low and large $Q$ is a measure of thickness of the shell and strain at the grain surface; (ii) the alp values measured at very large $Q$ tend to approach the value of the lattice parameter in the grain core; (iii) "jumps" of alp values calculated for individual reflections are caused by anisotropy of the grain shapes. ${ }^{9-11}$ The concept of alp has recently been used to analyze strains in nanocrystalline $\mathrm{SiC}$ powders. ${ }^{24}$

\section{RESULTS}

According to the manufacturer's specification for sample A more than $90 \%$ of carbon was in the form of $s p^{3}$ bonding and the rest was in the form of amorphous and graphitic carbon. High resolution TEM studies of nanodiamonds showed onionlike structures on the particle surfaces. ${ }^{25}$ Such structures could not affect the results of the x-ray analysis reported in this paper. All conclusions reached in this study are based on the analysis of diamond crystallographic lines. In the untreated samples B, C, and D there were no amorphous or graphitic structures. A negligible amount of graphite was noted after heat treatment at $1670 \mathrm{~K}$. At elevated temperatures diamond samples readily graphitize. ${ }^{25}$ The amount of graphite produced was estimated from the x-ray diffraction peak intensities. To minimize graphitization the outgassing was done at a relatively low temperature of $750 \mathrm{~K}$. Although compression was conducted at a higher temperature, special precautions such as the use of water-free talc reduced the amount of diamond transformed into graphite to less than $2 \%$.

The BET estimated particle size depends only on the surface area of the volume filled with diamond, whereas the data obtained from x-ray line broadening detect the coherently diffracting domain size. Thus, the ratio of the BET estimated particle size and the grain size obtained from the $\mathrm{x}$-ray line broadening could be interpreted as a number of coherently scattering crystalline domains per particle. The presence of amorphous carbon in sample A affects the estimated $d_{\mathrm{BET}}$ values listed in Table I. Considering 10\% to 20\% 
TABLE II. Microstructural parameters for diamond samples. Samples B were compressed at 2 and $5.5 \mathrm{GPa}$ at $1670 \mathrm{~K}$ for $60 \mathrm{~s}$. $\langle x\rangle_{\mathrm{vol}^{-}}$-volume-weighted mean crystallite size, $\sigma$ is the variance of the size distribution function, and $\rho$ is the density of dislocations.

\begin{tabular}{lcccc}
\hline \hline Sample & Pressure & $\langle x\rangle_{\text {vol }}$ & $\sigma$ & $\rho\left[10^{14} \mathrm{~m}^{-2}\right]$ \\
\hline A & $0.1 \mathrm{MPa}$ & $4(1)$ & $0.4(1)$ & $\leqslant 0.1$ \\
B & $0.1 \mathrm{MPa}$ & $16(3)$ & $0.8(1)$ & $3.0(5)$ \\
B & $2.0 \mathrm{GPa}$ & $22(3)$ & $0.6(1)$ & $6.2(5)$ \\
B & $5.5 \mathrm{GPa}$ & $22(3)$ & $0.6(1)$ & $6.4(5)$ \\
C & $0.1 \mathrm{MPa}$ & $24(3)$ & $0.9(1)$ & $1.4(2)$ \\
D & $0.1 \mathrm{MPa}$ & $66(8)$ & $1.1(1)$ & $1.6(2)$ \\
\hline \hline
\end{tabular}

error, it is likely that grains in samples A, B, and C consist of one crystallite, while grains of sample D consist of several coherently diffracting units.

The results of line-shape analysis of 12 diffraction peaks of diamond are listed in Table II. The data for sample A, as received, outgassed, and with different adsorbed species (nitrogen, water vapor, air) were identical and are not listed separately. For these smallest crystals it was difficult to assess concentration of dislocations. Dislocation concentration was very low, less than the sensitivity limit, $10^{13} \mathrm{~m}^{-2}$. The full width at half maximum (FWHM) of experimental line profiles for sample A are plotted in Fig. 3 as a function of $Q$. It is the so-called Williamson-Hall plot. Calculated FWHM values for $5 \mathrm{~nm}$ grain size diamond with stacking faults are also plotted in Fig. 3. The line broadening caused by stacking faults has been calculated by using DIFFAX, the software developed by Treacy et al. ${ }^{26}$ The measured FWHM values are slightly larger than the calculated ones, and the difference increases with $Q$ indicating that some microstrains were present in the particles, but in that case we were not able to quantify them.

The alp- $Q$ dependence of raw diamond samples of different grain sizes is depicted in Fig. 4. In Fig. 5, the alp- $Q$ data obtained for sample B processed at HPHT conditions are shown. Differences between the alp values for various samples reflect different ratio of surface to core atoms (Fig. 4) as well as changes of strains distribution in individual grains due to modification of their environment (Fig. 5).

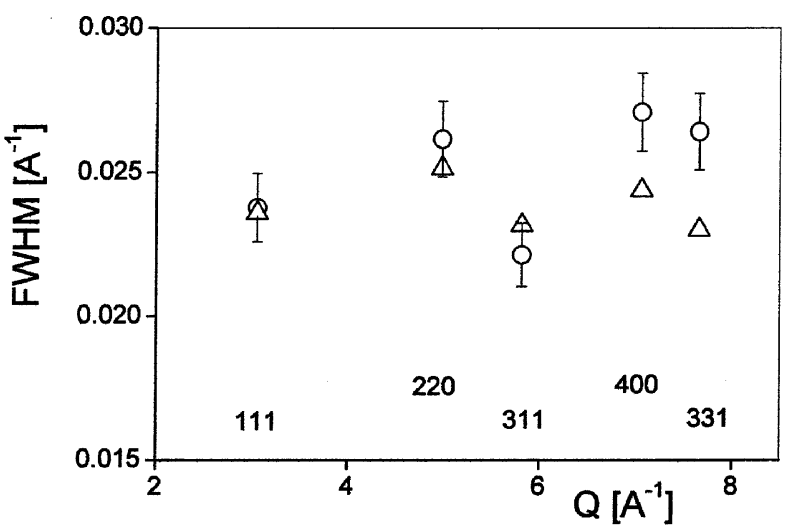

FIG. 3. Full width at half maximum (FWHM) of the line profiles as a function of $Q$ for specimen A (open circles). The calculated FWHM values for $5 \mathrm{~nm}$ diamond containing stacking faults are plotted as open triangles.

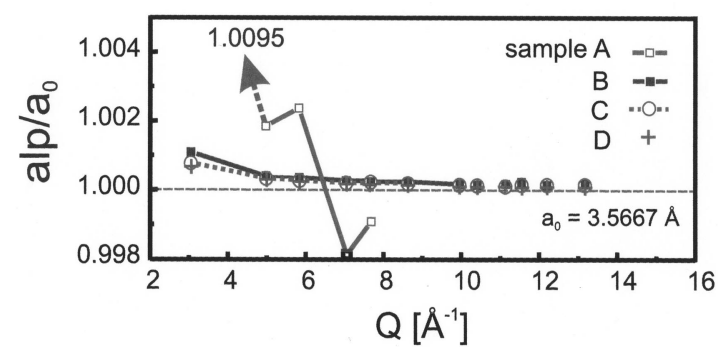

FIG. 4. alp- $Q$ plots for diamond powders of different nominal crystal sizes. Precision of the $a l p / a_{o}$ data is better than 0.0001 .

\section{DISCUSSION}

Increased alp values at low $Q$ indicate presence of an expanded shell structure. Such expansion is connected with strain development in the shell. It is reasonable to assume that specific structure and thickness of the surface shell is similar irrespective to size of the crystallite. For crystals of the smallest nominal sizes, $5 \mathrm{~nm}$, the relative number of atoms in the surface shell is very large, approximately $25 \%$ assuming $0.5 \mathrm{~nm}$ thick shell, but it is only $2 \%-3 \%$ for $50 \mathrm{~nm}$ size grains. The normalized alp value calculated for the (111) reflection of $5 \mathrm{~nm}$ grain powder is about $1 \%$ above $a_{0}$, but only about $0.1 \%$ for larger grain powders (see Fig. 4). This is understandable, with increasing size, the contribution of the shell atoms to the diffraction pattern decreases: The surface of grains larger than $20-30 \mathrm{~nm}$ becomes practically "invisible" for the diffraction experiment. For $50 \mathrm{~nm}$ and larger grains the measured $a l p$ values are very close to the lattice parameter of the interior of the crystal (Fig. 4). For samples $\mathrm{B}, \mathrm{C}$, and $\mathrm{D}$, the $a l p$ values in the whole $Q$ range differ very little and they are close to the lattice parameter of the grain core, $a_{0}$. For samples B, C, and D, the observed elevation of the alp values at low $Q$ can be explained in terms of the presence of very small grains, several nanometors in size. Indeed, the variance $\sigma$ for these samples obtained from the MWP procedure are large, see Table II, and consequently the particle size distribution functions are broad.

Irregular "jumps" in the alp- $Q$ plots observed for the $5 \mathrm{~nm}$ diamond powder are caused by anisotropy of the grains

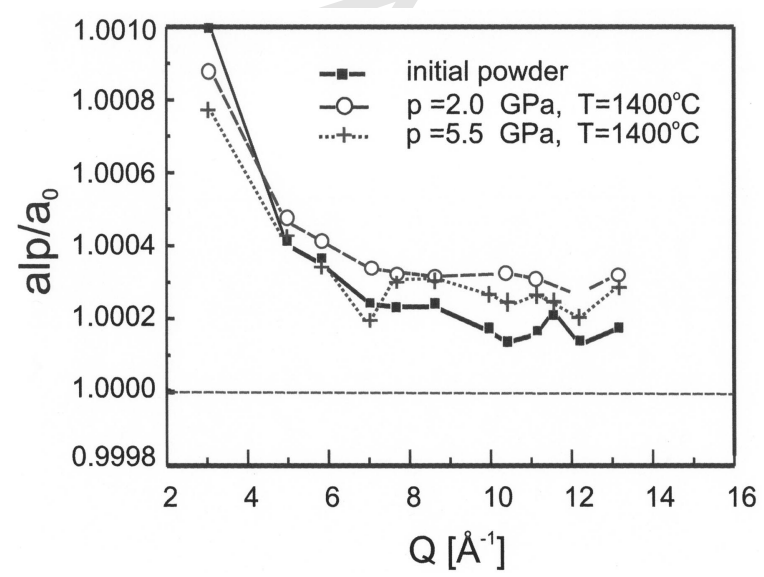

FIG. 5. alp- $Q$ dependence for diamond powder of nominal grain size $32 \mathrm{~nm}$, sample B, processed under HPHT conditions. Precision of the $a l p / a_{o}$ data is better than 0.0001 . 
shapes. ${ }^{11}$ This apparent size effect is most probably caused by stacking faults observed in anisotropic behavior of FWHM for the same specimen, as shown in Fig. 3. This effect is very weak for powders with large grains, B, C, D, which have more uniform shapes. Also the effect of grain shape on positions of Bragg reflections decreases with the increase of grain dimensions.

Annealing under vacuum reduces strains in the shell component and the alp values drop. ${ }^{11}$ Different surface reconstructions caused by adsorption of foreign atoms on the surface increase interatomic distances in the shell. However, the changes caused by adsorbed species are limited to the outer layers. No measurable changes in the diffraction line shapes indicated that the surface effects did not affect the population of defects, e.g., dislocations.

Evolution of the shell structure caused by heat treatment is pressure dependent. This is connected with the transformation of the free surface of individual powder grains in grain boundaries in the densified samples and simultaneous redistribution of strains between grain surface and core. The decrease in the alp values for low $Q$ is more pronounced for the sample treated at the higher pressure, sample B5.5 (Fig. $5)$. At the same time increased alp values for large $Q$ were observed, as compared to the initial, untreated powder. We speculate that the internal pressure in the grain interior is reduced when the surface strains relax. Similar effect was found for nanocrystalline $\mathrm{SiC} .{ }^{27}$ An additional outcome of that process, which increased strains in the core (increased alp at large $Q$ ), is greater population of dislocations, quantified by the MWP analysis. Dislocation density doubled as a result of heat treatment at external pressure of 2.0 and 5.5 GPa. These two conclusions clearly indicate that dislocations concentrate in the interior of the grains. This conclusion is further supported by the mentioned above analysis of the $5 \mathrm{~nm}$ diamond, sample A, after outgassing and after adsorption of various gasses. Outgassing and gas adsorption affected only the outer layers. ${ }^{11}$ This result is not surprising, as no dislocations are expected in grains smaller than $9 \mathrm{~nm}$.

\section{SUMMARY}

The apparent lattice parameter alp of the twocomponent, shell-core, model of nanosize diamond crystals and the multiple whole profile fitting analysis method were applied to analyze high resolution x-ray diffractograms of diamond powders of sizes varying between 5 and $250 \mathrm{~nm}$. For the $5 \mathrm{~nm}$ diamond powder the population of dislocations was small, less than the sensitivity of the method used, and it was impossible to evaluate their concentration. However, in larger particles presence of dislocations was clearly recognized and quantified. The population of dislocations increased during HPHT treatment while strains and interplanar distances in the shell decreased. Adsorption of foreign gasses on the grain surface modified the structure of the surface layers but left the interior of the grain and specifically the density of dislocations unaffected. The simultaneous application of the x-ray alp and line profile analysis methods provide complementary information about strains of different physical nature and origin. The method of alp tells us about the strains caused by surface reconstruction and/or relaxation, whereas, MWP procedure provides information about the strains produced by lattice defects, especially dislocations. First is more of homogeneous character, while, the latter is basically of inhomogeneous type.

\section{ACKNOWLEDGMENTS}

This project has been funded in part by the National Academy of Sciences under the Collaboration in Basic Science and Engineering Program supported by Contract No. INT-0002341 from the NSF. The contents of this publication do not necessarily reflect the views or policies of the National Academy of Sciences or the National Science Foundation, nor does mention of trade names, commercial products, or organizations imply endorsement by the National Academy of Sciences or the National Science Foundation. It was also supported in part by the EC Grant "Support for Centers of Excellence" No. ICA1-CT-2000-70005. J.G. and T.U. are grateful to the Hungarian National Science Foundation, OTKA, Grant Nos. F047057 and T046990. Thanks are due to K. Simon for his kind assistance in calculating the stacking fault effects in diamond.

${ }^{1}$ S. Veprek in Handbook of Ceramic Hard Materials, edited by R. Reidel (J. Wiley-VCH, Weinheim, Germany, 2000), pp. 104-139.

${ }^{2}$ C. P. Poole and F. J. Owens, Introduction to Nanotechnology (J. WileyInterscience, Hoboken, New Jersey, 2003).

${ }^{3}$ M. Winterer, Nanocrystalline Ceramics (Springer, Berlin, 2002).

${ }^{4}$ J.-Y. Raty, G. Galli, C. Bostedt, T. W. van Buuren, and L. J. Terminello, Phys. Rev. Lett. 91, 037401 (2003).

${ }^{5}$ S. H. Tolbert and A. P. Alivasatos, J. Phys. A 26, 56 (1993).

${ }^{6}$ Y. C. Lan, X. L. Chen, Y. P. Xu, Y. G. Cao, and F. Huang, Mater. Res. Bull. 35, 2325 (2000).

${ }^{7}$ Z. Chen and J. Ding, Nanostruct. Mater. 10, 205 (1998).

${ }^{8}$ L. Zhang, J. L. Coffer, T. W. Zerda, and W. Xu, Chem. Mater. 9, 2249 (1997).

${ }^{9}$ B. Palosz, E. Grzanka, S. Gierlotka, S. Stelmakh, R. Pielaszek, W. Lojnowski, U. Bismayer, J. Neuefeind, H.-P. Weber, and W. Palosz, Phase Transitions 76, 171 (2003).

${ }^{10}$ B. Palosz, E. Grzanka, S. Gierlotka, S. Stel'Makh, R. Pielaszek, U. Bismayer, J. Neuefeind, H. P. Weber, and W. Palosz, Acta Phys. Pol. A 102, 57 (2002).

${ }^{11}$ B. Palosz, E. Grzanka, S. Gierlotka, S. Stel'makh, R. Pielaszek, U. Bismayer, J. Neuefeind, H. P. Weber, T. Proffen, R. Von Dreele, and W. Palosz, Z. Kristallogr. 217, 497 (2002).

${ }^{12}$ I. E. Reimanis, Mater. Sci. Eng., A 237, 159 (1997).

${ }^{13}$ Y. Zhao, J. Qian, L. Daemen, C. Pantea, J. Zhang, and T. W. Zerda, Appl. Phys. Lett. (to be published).

${ }^{14}$ T. Ungar, J. Gubicza, G. Ribarik, and A. Borbely, J. Appl. Crystallogr. 34, 298 (2001)

${ }^{15}$ C. Pantea, J. Gubicza, T. Ungar, G. A. Voronin, and T. W. Zerda, Phys. Rev. B 66, 094106 (2002).

${ }^{16}$ T. Ungár, I. Dragomir, Á. Révész, and A. Borbély, J. Appl. Crystallogr. 32, 992 (1999).

${ }^{17}$ J. Qian, C. Pantea, G. A. Voronin, and T. W. Zerda, J. Appl. Phys. 90, 1632 (2001).

${ }^{18}$ L. G. Khvostantsev, L. F. Vereshchagin, and A. P. Novikov, High Temp. High Press. 9, 637 (1977).

${ }^{19}$ M. Wilkens, in: Fundamental Aspects of Dislocation Theory, Vol. II (US National Bureau Standards, Special Publication No. 317, edited by J. A. Simmons, R. de Wit, and R. Bullough, Washington, DC, 1970), p. 1195.

${ }^{20}$ M. Wilkens, Phys. Status Solidi A 2, 359 (1970).

${ }^{21}$ Z. Kaszkur, J. Appl. Crystallogr. 33, 1262 (2000).

${ }^{22}$ C. W. B. Grogson and E. Barton, J. Appl. Phys. 18, 175 (1967).

${ }^{23}$ D. Zanchet, B. D. Hall, and D. Ugarte, in Characterization of Nanophase Materials, edited by Z. L. Wang (J. Wiley-VCH, Weinheim, Germany, 2000), pp. 13-36.

${ }^{24}$ B. Palosz, S. Stel'makh, E. Grzanka, S. Gierlotka, R. Pielaszek, U. Bis- 
mayer, S. Werner, and W. Palosz, J. Phys.: Condens. Matter 16, S353 (2004).

${ }^{25}$ J. Qian, Ph.D. dissertation, TCU, Fort Worth, 2003.

${ }^{26}$ M. M. J. Treacy, J. M. Newsam, and M. W. Deem, Proc. R. Soc. London,
Ser. A 433, 499 (1991).

${ }^{27}$ S. Stel'makh, S. Gierlotka, E. Grzanka, H.-P. Weber, and B. Palosz, J. Alloys Compd. 382, 138 (2004).

\section{PROOF COPY 089505JAP}

